\title{
Model of the Effects of Improving TB Diagnosis on Infection Dynamics in Differing Demographic and HIV-Prevalence Scenarios
}

\author{
Allison S. Rhines ${ }^{1 *}$, Midori Kato-Maeda ${ }^{2}$, Marcus W. Feldman ${ }^{1}$ \\ ${ }^{1}$ Department of Biology, Stanford University, Stanford, USA \\ ${ }^{2}$ Curry International Tuberculosis Center, Division of Pulmonary and Critical Care Medicine, University of \\ California, San Francisco, USA \\ Email: *arhines@stanford.edu, Midori.Kato-Maeda@ucsf.edu, mfeldman@stanford.edu
}

Received 5 January 2015; accepted 29 January 2015; published 30 January 2015

Copyright (C) 2015 by authors and Scientific Research Publishing Inc.

This work is licensed under the Creative Commons Attribution International License (CC BY).

http://creativecommons.org/licenses/by/4.0/

(c) (i) Open Access

\begin{abstract}
This paper seeks to examine the sensitivity of tuberculosis transmission (TB) dynamics to the rate at which infectious individuals with active TB begin a TB treatment course, and therefore cease to be infectious to others. We model this by varying both the rate at which individuals are diagnosed and begin treatment, and the demographic conditions in which the epidemic occurs. An age-structured deterministic ordinary differential equation model is used to study the sensitivity of TB transmission dynamics to the implementation of a more effective diagnostic such as Xpert MTB/ RIF in a high HIV prevalence setting. Sensitivity analysis of the effectiveness of the diagnostic $(\lambda)$ shows the interim disease dynamics in three demographic scenarios defined by differences in HIV prevalence and age structure at a constant transmission rate. In the near future, we expect the diagnostic to have the most effect in areas of high HIV prevalence. In the long term, we expect the diagnostic to have the most significant impact at high transmission rates regardless of HIV prevalence and age structure.
\end{abstract}

\section{Keywords}

Tuberculosis, Diagnostics, Xpert MTB/RIF, TB/HIV Co-Infection, Demography

\footnotetext{
*Corresponding author.
}

How to cite this paper: Rhines, A.S., Kato-Maeda, M. and Feldman, M.W. (2015) Model of the Effects of Improving TB Diagnosis on Infection Dynamics in Differing Demographic and HIV-Prevalence Scenarios. Journal of Tuberculosis Research, 3, 1-10. http://dx.doi.org/10.4236/jtr.2015.31001 


\section{Introduction}

To eliminate tuberculosis (TB), a reduction in incidence is imperative. One of the strategies to reduce the incidence of TB is to effectively treat all patients with TB with early case detection and higher cure rates, which will interrupt transmission [1].

Time to treatment could be decreased through the use of a more accurate diagnostic, by reducing time to diagnosis, or other interventions access to care once diagnosed.

There is opportunity for improvement in commonly used TB diagnostics, both in accuracy and speed of diagnosis. Sputum smear microscopy and culture have to date been the most common methods to diagnose TB disease in the developing world. Sputum smear examination ranks poorly by most standards of diagnostic accuracy, even in the developing world, with sensitivity varying from $32 \%$ to $97 \%$ and specificity from $94 \%$ to $100 \%$ when using culture as a gold standard [2]. Although culture is the most sensitive method, it is not only expensive, and can take more than four weeks to provide a result [3], but also requires training and sophisticated laboratory equipment, which are often not available in parts of the developing world [4].

In recent years, there have been major advances in methods of TB diagnostic technology. In particular, nucleic acid amplification test (NAAT) methodology, has led to significant improvements over smear microscopy in areas where diagnosis by culture is not feasible. One prominent example is Xpert MTB/RIF (GeneXpert system; Cephid, Sunnyvale, California), a NAAT diagnostic employing fully automated, cartridge-based testing [5] has been shown to have a better sensitivity (88\%) and specificity (98\%) than sputum smear microscopy [6] and to be almost as accurate as culture. Xpert MTB/RIF is particularly notable for its ability to detect TB in HIV positive individuals, and to detect extra-pulmonary TB, both of which are extremely difficult to identify with conventional methods [7]. Moreover, Xpert MTB/RIF has a significantly reduced time to detection of TB compared with sputum smear microscopy, and culture on solid or liquid media [8]. In addition to its improved accuracy over sputum smear microscopy, Xpert MTB/RIF facilitates the logistics of TB testing in ways that could potentially lead to the performance of more tests. Xpert MTB/RIF operates under comparatively low biohazard levels, by using all self-contained, sealed cartridges employing a fully automated system [9] [10]. The agreement to reduce its cost to 9.98 USD in 145 high burden countries [11] may also make Xpert MTB/RIF the most cost-effective option to diagnose TB.

While a next generation of TB tests will no doubt build on these technological improvements, study of the effects of these improvements on the transmission dynamics and epidemic trajectory of TB remains limited. A model studying the effects of Xpert MTB/RIF on TB transmission [12], suggested that its effects on epidemic dynamics will be important. However, this particular model was designed and parameterized with the Indian TB epidemic specifically in mind; countries with higher HIV prevalence and significantly different demographic structure may experience different changes in disease dynamics as a result of a more effective diagnostic.

While India has the largest absolute number of TB cases due to its large population, the highest per capita prevalence of TB is in Sub-Saharan Africa [13]. The Sub-Saharan African TB epidemic, unlike the Indian epidemic, is characterized by high rates of TB-HIV co-infection [14]. Additionally, Sub-Saharan African population structure is characterized by high HIV-related mortality among adults, and high fertility, which have been factors in skewing the Southern African age structure, with over a third of the population younger than age 15 in some countries [15]-[17].

Age structure and HIV prevalence are potentially relevant to TB dynamics because of the ways in which TB is transmitted. While HIV positive individuals and children can contract the disease, they are significantly less likely to transmit TB to their contacts [18] [19]. TB is typically transmitted when cavitation occurs in the lungs, allowing bacteria to be expelled by coughing, though exceptions have been shown [20]. Children with tuberculosis do not usually have severe lung lesions and harbor fewer bacteria than adults, causing them to transmit at lower rates [21]. Patients who are HIV-positive have a range of clinical manifestations of TB. If their immune systems are relatively conserved, the symptoms are similar to those of people not infected with HIV. However, if their immune system is compromised, the disease can be disseminated [22] [23]. If children and HIV-positive adults are assumed to be minimally infectious to others [21]-[23], a model that takes account of the potential effects of an improved diagnostic within populations of different age structure and HIV prevalence would be useful in assessing its potential impact on TB transmission dynamics in Sub-Saharan Africa.

Recently, Lin et al. evaluated the impact of Xpert MTB/RIF in Sub-Saharan Africa. They used a model taking account of the demographic and HIV prevalence context in which Xpert MTB/RIF or similar diagnostic would 
be used in Tanzania [24]. The authors predicted that the effect of such a diagnostic is likely to be significant in this high HIV prevalence area, projecting that while use of sputum smear microscopy in these areas will lead to a reduction of $3.94 \%$ in new TB cases per year, a diagnostic method such as Xpert MTB/RIF could lead to a 4.25\% reduction per year in Tanzania. Their conclusion suggests that HIV, demographic structure, and other contextual factors are likely to influence the impact of such an intervention. Menzies et al. modeled the cost effectiveness of Xpert MTB/RIF in Sub-Saharan African countries, concluding that it will likely be a cost effective intervention, leading to significant improvements in mortality and morbidity in the short term, and some effects in the long term [25].

In this manuscript, we analyze the sensitivity of TB infection dynamics to the rate at which infectious individuals with active TB are placed on a TB treatment course, and therefore cease to be infectious to others. We create a model to explore how improving TB diagnostic effectiveness and therefore reducing time to treatment for infectious individuals, may influence epidemiological dynamics in demographic context. Study of such a dynamic system of infectious TB is timely, as a relatively novel set of molecular diagnostics has great potential to affect the rate at which individuals are placed on TB treatment. The availability of a diagnostic allowing more rapid detection of TB is potentially relevant to transmission dynamics because, once individuals are aware that they are infected, they can begin treatment, thereby ceasing to be infectious to others.

We model a range of potential effects of increasing the rate at which individuals with active infectious TB are placed on treatment, causing them to cease transmitting TB to others, in a variety of African demographic contexts. Such perturbations in the TB dynamic system could arise due to increased use of rapid NAAT diagnostic. We studied these effects on the trajectory of a hypothetical TB epidemic in a variety of demographic settings as described below.

\section{Methods}

We studied the sensitivity of TB infection dynamics to the rate at which infectious individuals with active TB are placed on a TB treatment course, and therefore cease to be infectious to others, by varying both 1 ) the rate at which individuals are diagnosed and begin TB treatment, and 2) the demographic conditions in which the epidemic occurs, to reflect the diverse demographic scenarios in Sub-Saharan Africa and to explore influence of demography. We use a closed-population ordinary differential equation model adapted from Modeling Infectious Diseases in Humans and Animals [26] to describe TB disease dynamics, with modifications to include HIV status and age structure. Sensitivity analysis was performed by varying the parameter associated with diagnostic accuracy and efficiency to understand the range of effects of an improved diagnostic may have on the epidemic trajectory of TB.

\subsection{Demographic Structure}

We simulated the epidemic under three different sets of demographic conditions similar to those in diverse areas of Africa. Age structure was maintained throughout the simulation even though HIV and TB infections modulate HIV and TB status categories of individuals within an age group. All individuals aged 14 and younger were categorized as children.

Demographic scenarios are described in Table 1: Demographic Scenarios. Demographic Scenario 1 is one of a moderately young population (28.3\% age less than 14) and high HIV prevalence (17.8\%), designed to reflect modern South Africa. Scenario 2 is designed to study the epidemic in a demographic regime featuring very young age structure (44.8\% aged less than 14$)$, and moderate HIV prevalence (5.6\%), using modern Tanzania as a guide. Scenario 3 features very young age structure (36.9\% aged less than 14 ) and high HIV prevalence (25.9\%), based approximately on modern Swaziland [27]. These demographic scenarios are selected only as examples for the sake of comparison, and study of the influence of demography. Because TB rates are standardized across scenarios to facilitate comparison, they do not produce actual predictions for the future of TB in the countries represented by the scenarios.

\subsection{Variables}

Table 2: Variables describes the breakdown of the population by age and disease status. There are three demographic categories of individuals: child, HIV-negative adult and HIV-positive adult. We distinguish children and 
Table 1. Demographic scenarios.

\begin{tabular}{cccc}
\hline Country Basis for Scenario & Population Aged < 14 (\%) & HIV+ Adults (\%) & HIV- Adults (\%) \\
\hline South Africa (Demographic Scenario 1) & 28.3 & 17.8 & 53.9 \\
Tanzania (Demographic Scenario 2) & 44.8 & 5.6 & 49.6 \\
Swaziland (Demographic Scenario 3) & 36.9 & 25.9 & 37.2 \\
\hline
\end{tabular}

Table 2. Variables.

\begin{tabular}{|c|c|c|}
\hline & Variables & Description \\
\hline \multirow{3}{*}{ Children } & $\mathrm{S}_{\text {Child }}$ & Children susceptible to TB \\
\hline & $\mathrm{L}_{\text {Child }}$ & Children with latent TB \\
\hline & A $_{\text {Child }}$ & Children with active TB \\
\hline \multirow{4}{*}{ HIV- Adults } & $\mathrm{S}_{\text {HIV- }}$ & HIV- Adults susceptible to TB \\
\hline & LHIV- & HIV- Adults with latent TB \\
\hline & $\mathrm{H}_{\text {HIV- }}$ & HIV- Adults with active infectious TB \\
\hline & $\mathrm{A}_{\text {HIV- }}$ & HIV- Adults with active TB \\
\hline \multirow{3}{*}{ HIV+ Adults } & $\mathrm{S}_{\mathrm{HIV}+}$ & HIV+ Adults susceptible to TB \\
\hline & $\mathrm{L}_{\mathrm{HIV}+}$ & HIV+ Adults with latent TB \\
\hline & $\mathrm{A}_{\mathrm{HIV}+}$ & HIV+ Adults with active TB \\
\hline
\end{tabular}

Legend: All variables in the model are listed by age, HIV status and TB status. The S category refers to individuals susceptible to TB, L to those who are latently infected, $\mathrm{H}$ to those who are actively infected and infectious to others, and A to those who are actively infected but not infectious to others.

HIV-positive individuals from HIV negative adults in the transmission model, as both of these groups are susceptible to TB, however, neither transmits the disease at significant rates to their contacts [21]-[23]. HIV-negative adults are thus considered to be the only transmitters of TB. Therefore within the groups of children and HIVpositive adults, an individual can be: susceptible, infected with latent TB, or infected with active TB. Within the group of HIV-negative adults, an individual may also be infected with active infectious TB. The structure of the model is detailed in the Model Structure section.

\subsection{Parameters}

We performed sensitivity analysis to study the effect of perturbing the rate at which individuals move from the infected and infectious class to the infected only class, $\lambda$. This move occurred when the individual commences TB treatment. A new diagnostic method such as Xpert MTB/RIF differs from existing methods in ways that might decrease time to treatment due to improved logistics surrounding testing procedure and accuracy, such as: 1) the likelihood of and delay in being tested [28]; 2) the accuracy (sensitivity and specificity) of the test [29]; 3) time to treatment once a positive diagnosis is reached [3]. The parameter $\lambda$ represents with a single parameter combination of these and other ways in which an improved diagnostic might influence TB transmission. This parameter should be thought of as a multiplier to represent the proportion of diseased individuals who move into the treated category per unit time, that is, from the infected and infectious category $\left(\mathrm{H}_{\mathrm{HIV}-}\right)$ to the infected category $\left(\mathrm{A}_{\mathrm{HIV}-}\right)$. For the purpose of the model, we assume that only HIV-individuals transmit.

TB parameters for the simulation are described in Table 3 . These are estimated, or based on the literature if available [30]. Other parameters are listed in Table 4. Their values are selected to maintain constant population structure, that is, constant proportions of the population in each demographic group.

\subsection{Model Structure}

The mathematical model of the TB epidemic in which new NAAT diagnostics would be implemented is depicted 
Table 3. TB parameters.

\begin{tabular}{ccccc}
\hline & TB Parameters & Description & Selected Value & $\begin{array}{c}\text { Value/Population Size } \\
(=100)\end{array}$ \\
\hline \multirow{2}{*}{ General } & $\beta$ & Transmission parameter & 10 & 0.1 \\
& $\rho$ & Proportion of population that immediately develop & 5 & 0.05 \\
active TB upon infection & Progression rate to active TB & 5 & 0.05 \\
Children & $\eta$ & TB recovery rate & 90 & 0.9 \\
HIV- & $\tau$ & Progression rate to active infectious TB & 5 & 0.05 \\
& $\lambda$ & Rate at which individuals with active & Sensitivity & Sensitivity analysis \\
HIV + & $\gamma$ & TB infections access treatment & 90 & 0.9 \\
\hline
\end{tabular}

Legend: This table lists TB infection parameters in our model. All parameters are in units of cases per year.

\section{Table 4. HIV and demographic parameters.}

\begin{tabular}{|c|c|c|c|}
\hline $\begin{array}{l}\text { HIV and Aging } \\
\text { Parameters }\end{array}$ & Description & $\begin{array}{l}\text { Value/Population } \\
\text { Size }(=100)\end{array}$ & Rationale \\
\hline $\mathrm{a}$ & $\begin{array}{l}\text { Rate at which children susceptible to TB become } \\
\text { HIV+ TB-susceptible adults }\end{array}$ & $6.15 \mathrm{E}-05$ & $\begin{array}{l}\text { Aging rate to maintain constant } \\
\text { population structure } \times \text { childhood } \\
\text { incidence of } \mathrm{HIV}\end{array}$ \\
\hline $\mathrm{b}$ & $\begin{array}{c}\text { Rate at which children susceptible to TB become } \\
\text { HIV-adults susceptible to TB }\end{array}$ & 0.071424179 & $\begin{array}{l}\text { Aging rate to maintain constant } \\
\text { population structure } \times \\
\text { (1-childhood incidence of TB) }\end{array}$ \\
\hline $\mathrm{C}$ & $\begin{array}{l}\text { Rate at which HIV- adults susceptible to } \\
\text { TB become HIV+ adults susceptible to TB }\end{array}$ & 0.003234425 & $\begin{array}{c}\text { Adult HIV incidence } \\
\text { (new HIV cases per year/population size) }\end{array}$ \\
\hline $\mathrm{d}$ & $\begin{array}{l}\text { Rate at which children with latent } \\
\text { TB become HIV+ adults with latent TB }\end{array}$ & $6.15 E-05$ & $\begin{array}{l}\text { Aging rate to maintain constant population } \\
\text { structure } \times \text { childhood incidence of HIV }\end{array}$ \\
\hline $\mathrm{e}$ & $\begin{array}{l}\text { Rate at which children with latent } \\
\text { TB become HIV- adults with latent TB }\end{array}$ & 0.071424179 & $\begin{array}{c}\text { Aging rate to maintain constant } \\
\text { population structure } \times \\
\text { (1-childhood incidence of TB) }\end{array}$ \\
\hline $\mathrm{f}$ & $\begin{array}{l}\text { Rate at which HIV- adults with latent } \\
\text { TB become HIV+ adults with latent TB }\end{array}$ & 0.003234425 & $\begin{array}{c}\text { Adult HIV incidence } \\
\text { (new HIV cases per year/population size) }\end{array}$ \\
\hline g & $\begin{array}{c}\text { Rate at which children with active TB become } \\
\text { HIV+ adults with active TB }\end{array}$ & $6.15 \mathrm{E}-05$ & $\begin{array}{l}\text { Aging rate to maintain constant population } \\
\text { structure } \times \text { childhood incidence of HIV }\end{array}$ \\
\hline $\mathrm{h}$ & $\begin{array}{l}\text { Rate at which children with active } \\
\text { TB become HIV- adults with active TB }\end{array}$ & 0.035712089 & $\begin{array}{l}\text { Aging rate to maintain constant population } \\
\text { structure } \times(1 \text {-childhood incidence of TB)/2 }\end{array}$ \\
\hline $\mathrm{i}$ & $\begin{array}{l}\text { Rate at which HIV- adults with active } \\
\text { TB become HIV+ adults with active TB }\end{array}$ & 0.003234425 & $\begin{array}{c}\text { Adult HIV incidence } \\
\text { (new HIV cases per year/population size) }\end{array}$ \\
\hline j & $\begin{array}{l}\text { Rate at which adults with HIV- adults with active } \\
\text { infectious TB become HIV+ adults with active TB }\end{array}$ & 0.003234425 & $\begin{array}{c}\text { Adult HIV incidence } \\
\text { (new HIV cases per year/population size) }\end{array}$ \\
\hline $\mathrm{k}$ & $\begin{array}{l}\text { Rate at which children with active TB become } \\
\text { adults with active infectious TB }\end{array}$ & 0.035712089 & $\begin{array}{l}\text { Aging rate to maintain constant } \\
\text { population structure } \times \\
\text { (1-childhood incidence of TB)/2 }\end{array}$ \\
\hline $\mathrm{x}$ & HIV mortality rate & 0.05 & $\begin{array}{l}\text { Assumed mortality set to } \\
\text { maintain constant population }\end{array}$ \\
\hline $\mathrm{y}$ & TB mortality rate & 0.08 & $\begin{array}{l}\text { Assumed mortality set to } \\
\text { maintain constant population }\end{array}$ \\
\hline$\mu$ & Background mortality rate & 0.03 & $\begin{array}{l}\text { Assumed mortality set to } \\
\text { maintain constant population }\end{array}$ \\
\hline
\end{tabular}

Legend: This table lists HIV and demographic parameters in the model. 
in Figure 1: Model Structure. The general framework of the model is as below. We focused exclusively on the effects of these diagnostics concerning drug-susceptible TB dynamics, ignoring the additional capacity of Xpert MTB/RIF to detect Rifampin resistance. The time units of the model are years. The full mathematical model depicted in Figure 1: Model Structure is presented in Equations.

The model is designed to explore the potential effects of improved time to treatment though more accurate and timely diagnosis. TB and HIV infection dynamics are considered independently. In the model, all children and HIV+ adults can be susceptible to TB, latently infected with TB, or actively infected but not infectious to others. HIV - adults can be in any of these TB disease states; additionally, they can be infected with active TB, and, before they are placed on treatment, can be actively infected and infectious to others. We explore the effects of changing the rate at which these individuals move from the infected and infectious, to the infected and noninfectious compartment on TB infection dynamics, an effect that may be accomplished though improved diagnostics.

TB infection is governed by the rate of interaction between those susceptible to TB and those infected with active, infectious TB, and the infection parameter, $\beta$. A fraction of those individuals who become infected immediately develop active TB at the time they become infected $(\rho)$; the remainder of those infected develop latent TB. Individuals recover from active TB at rates $\gamma$ (HIV-), $\alpha$ (HIV+), and $\tau$ (children). HIV infection and aging dynamics are modeled separately, with individuals moving between TB, HIV and age states independently. There is mortality in every disease state.

\section{Results and Discussion}

\subsection{Interim TB Dynamics}

Sensitivity analysis of the effectiveness of the diagnostic $(\lambda)$ showed the interim disease dynamics in three de-

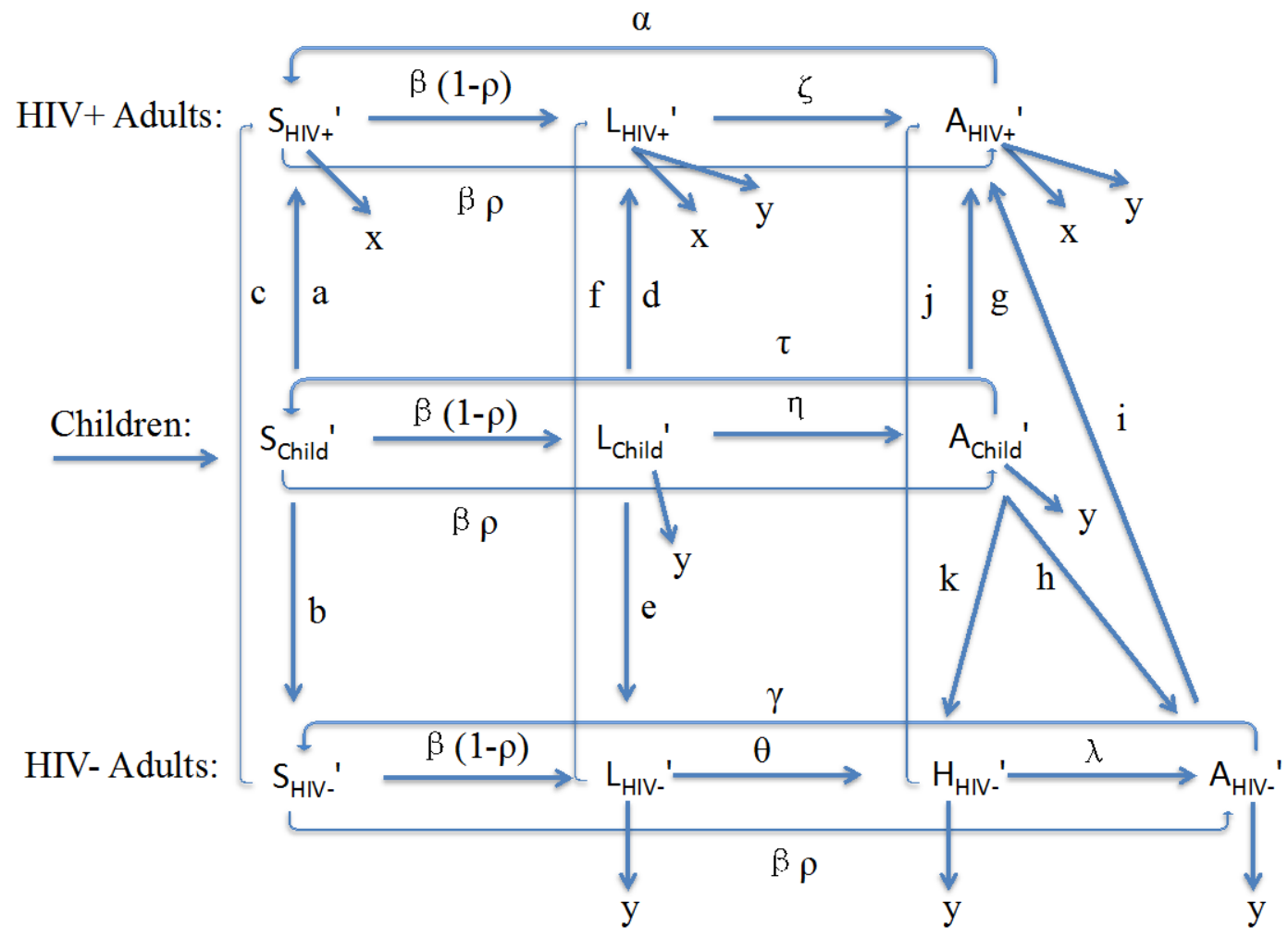

Figure 1. Model Structure. Legend: This figure represents the model used to explore the potential impact of improved diagnostics on TB transmission. In the model, individuals can be infected independently with TB and/or HIV and can transition between age classes. Individuals begin as children, HIV+ adults or HIVadults. Individuals within all of these categories can be susceptible to TB, latently infected with TB, or actively infected with TB. Only HIV- individuals infected with active TB (HHIV-) can transmit TB to others. 
mographic scenarios considering differences in HIV prevalence and age structure with a constant TB transmission rate. Plots of sensitivity analysis concerning TB transmission rate and effectiveness of the diagnostic are included in Supplementary Materials.

The infectious dynamics of the TB epidemic changed in response to population structure as well as to diagnostic effectiveness. Within every level of diagnostic effectiveness $\lambda$, varying the demographic context resulted in differing dynamics throughout the course of the epidemic. Most notable is the case of the highest HIV prevalence setting for example (Scenario 3), where the proportion of susceptible HIV-adults initially peaked before decreasing considerably in favor of latent infections, as in the other demographic settings. The differences in disease dynamics between these simulations occur in the timeframe in which the diagnostic is most likely be evaluated (within the first 20 years).

Change in disease dynamics was also observed by varying the effectiveness of the diagnostic $(\lambda)$ within a given demographic context. Within every demographic scenario, the delay in growth in the proportion of active infectious individuals is increased with additional diagnostic effectiveness. That is, though the equilibrium case values are not significantly different, the rate at which the equilibrium is reached changes significantly with the power of the diagnostic, which has implications for its implementation.

\subsection{Equilibrium TB Prevalence}

Sensitivity analysis of the number of active cases when the simulation has reached equilibrium to variance in the transmission rate $(\beta)$ and the effectiveness of the diagnostic $(\lambda)$ is shown in Figure 2: Equilibrium Active Cases. Independent of demographic conditions, the prevalence of TB appears to converge to this distribution given the ranges of the transmission rate $(\beta)$ and the effectiveness of the diagnostic $(\lambda)$.

Though the simulation does not reach equilibrium until after the implementation timeframe for the diagnostic, from Figure 2: Equilibrium Active Cases, we can observe the combined effects of varying diagnostic effectiveness and transmission probability on the equilibrium outcome. Most notable, for a given value of the diagnostic parameter $(\lambda)$, that the effectiveness of the diagnostic or lack thereof $(\lambda)$, we can observe from the large variance in ending case numbers for high values of the transmission rate $(\beta)$ compared to low values has the greatest effect on equilibrium prevalence when the transmission rate $(\beta)$ is greatest.

\subsection{Related Work and Areas for Future Research}

Our results are novel in the sense that they explicitly explore the relationship between demographic conditions and the potential for improved TB diagnosis, however they appear to be generally consistent with other work that has been completed in the area of NAAT diagnostic modeling for TB. The focus of our model on the shortterm benefits of such diagnostics is consistent with the predicted short-term benefits predicted by Menzies et al. [25]. Our results also support the conclusions of Lin et al. that consideration of context is key in scaling up diagnostics [24], though we limit our consideration of context to demographic context. Our exploration of the potential to avert cases by curbing transmission responds to limitations in other studies; future research will be needed to fully explore the epidemiological and cost-effectiveness consequences of such interventions.

One limitation of our work is that it does not separate the many aspects of diagnostic effectiveness from each other, including access to the diagnostic, technical sensitivity of the diagnostic, and likelihood of starting and completing and treatment following the diagnosis of TB. Future studies should examine the roles of these factors independently. The role of more granular data will also be key in making estimates of the potential effectiveness of new interventions in specific areas. Future models of the interactions between demography and infectious disease intervention implementation designed to make accurate predictions for specific countries may also consider the effects of realistic population aging, rather than constant age structure.

A final key area for future study will be the role of broader societal factors in effective TB diagnosis. The work of Evans, for example, suggests that active case finding will be essential in maximizing the benefits of NAAT diagnostics. As cases cannot even be diagnosed if they do not present to clinicians, the influence of socioeconomic factors underlying access to care will undoubtedly be important in interrupting the chain of transmission though effective treatment [31]. However, Small and Pai highlight the possibilities arising from a "virtuous cycle” beginning with improved diagnostic tests, and leading to improvement in other aspects of TB care [10]. 


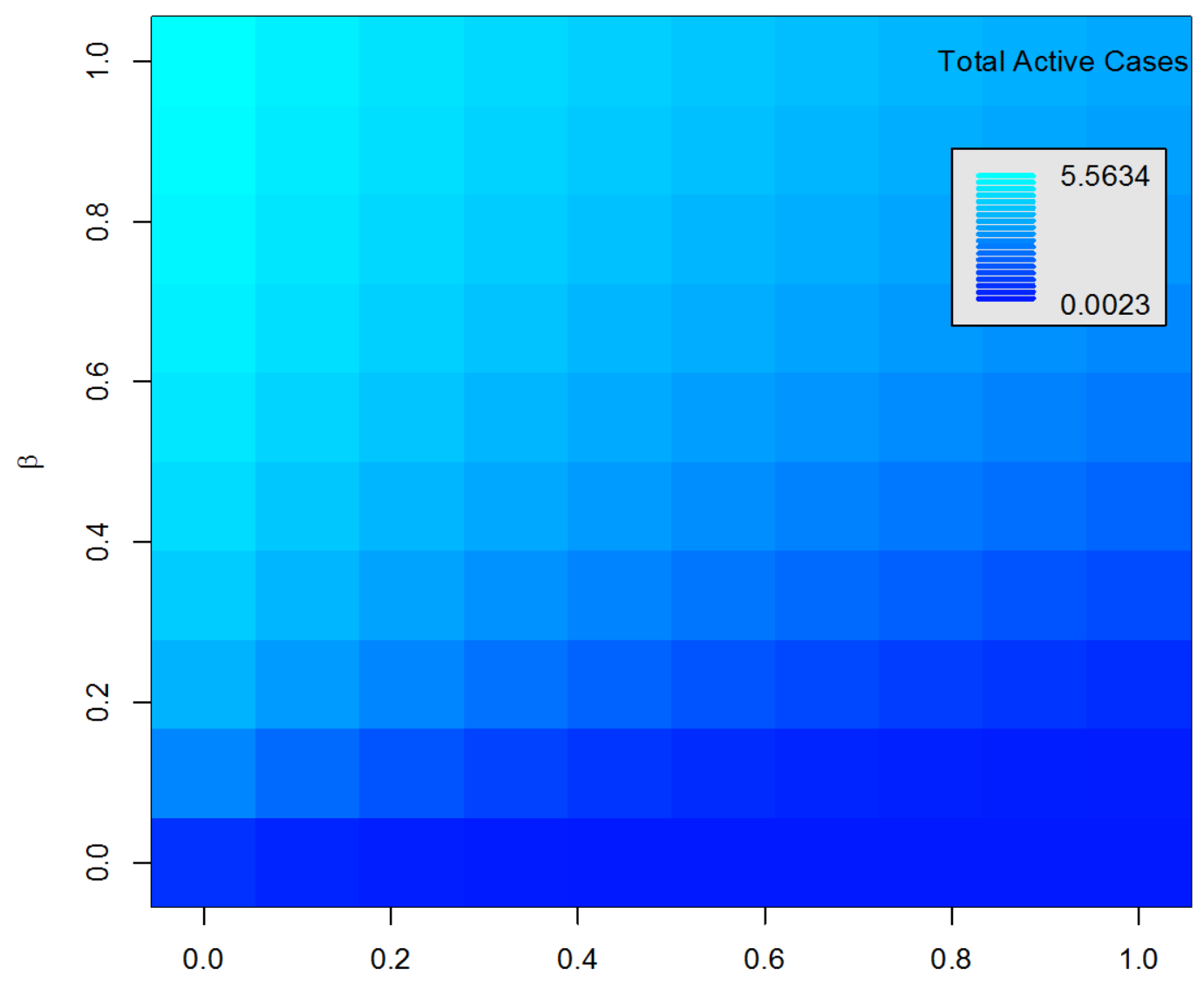

Figure 2. Equilibrium Active Cases. Legend: Sensitivity analysis of the number of cases of active TB at equilibrium to variance in the transmission rate $(\beta)$ and the effectiveness of the diagnostic $(\lambda)$. The data shown is generated using Scenario 1, though the model appears to convergence on this set of values.

\section{Conclusion}

The demographic context in which a diagnostic is introduced is shown to affect the trajectory of the epidemic, and therefore, the success of the diagnostic intervention. This effect is visible in the simulations independent of the level of effectiveness of the diagnostic intervention. We conclude that demographic context should be accounted for in the introduction of a new diagnostic intervention designed to increase the proportion of individuals with TB disease who receive treatment such as Xpert MTB/RIF. Public health interventions to expand the introduction of novel TB diagnostics should recognize that the disease dynamics following the introduction of such a diagnostic may be subject to HIV prevalence and age structure of the population in question. Though epidemiological studies will be needed to confirm and clarity these impacts, this model suggests a need for such future studies.

\section{Acknowledgements}

We would like to acknowledge Dr. Nicole Creanza for assistance with model implementation and data analysis. This material is based upon work supported by the National Science Foundation Graduate Research Fellowship under Grant No. DGE-114747.

\section{References}

[1] Dye, C., Glaziou, P., Floyd, K. and Raviglione, M. (2013) Prospects for Tuberculosis Elimination. Annual Review of Public Health, 34, 271-286. http://www.ncbi.nlm.nih.gov/pubmed/23244049 http://dx.doi.org/10.1146/annurev-publhealth-031912-114431

[2] Boehme, C.C., Nicol, M.P., Nabeta, P., Michael, J.S., Gotuzzo, E., et al. (2011) Feasibility, Diagnostic Accuracy, and 
Effectiveness of Decentralised Use of the Xpert MTB/RIF Test for Diagnosis of Tuberculosis and Multidrug Resistance: A Multicentre Implementation Study. Lancet, 377, 1495-1505.

http://www.pubmedcentral.nih.gov/articlerender.fcgi?artid=3085933\&tool=pmcentrez\&rendertype=abstract http://dx.doi.org/10.1016/S0140-6736(11)60438-8

[3] Millen, S.J., Uys, P.W., Hargrove, J., van Helden, P.D. and Williams, B.G. (2008) The Effect of Diagnostic Delays on the Drop-Out Rate and the Total Delay to Diagnosis of Tuberculosis. PLoS One, 3, e1933.

http://www.pubmedcentral.nih.gov/articlerender.fcgi?artid=2276686\&tool=pmcentrez\&rendertype=abstract

[4] Perkins, M.D. and Cunningham, J. (2007) Facing the Crisis: Improving the Diagnosis of Tuberculosis in the HIV Era. The Journal of Infectious Diseases, Suppl 196, S15-S27. http://www.ncbi.nlm.nih.gov/pubmed/17624822 http://dx.doi.org/10.1086/518656

[5] Tortoli, E., Russo, C., Piersimoni, C., Mazzola, E., Dal, M.P., et al. (2012) Clinical Validation of Xpert MTB/RIF for the Diagnosis of Extrapulmonary Tuberculosis. European Respiratory Journal, 1-14. http://www.ncbi.nlm.nih.gov/pubmed/22241741

[6] Steingart, K., Sohn, H., Schiller, I., Kloda, L., Boehme, C., et al. (2013) Xpert ${ }^{\circledR}$ MTB/RIF Assay for Pulmonary Tuberculosis and Rifampicin Resistance in Adults. Cochrane Database of Systematic Reviews, Issue 1. http://dx.doi.org/10.1002/14651858.CD009593.pub2

[7] Hillemann, D., Rüsch-Gerdes, S., Boehme, C. and Richter, E. (2011) Rapid Molecular Detection of Extrapulmonary Tuberculosis by the Automated GeneXpert MTB/RIF System. Journal of Clinical Microbiology, 49, 1202-1205. http://www.pubmedcentral.nih.gov/articlerender.fcgi?artid=3122824\&tool=pmcentrez\&rendertype=abstract http://dx.doi.org/10.1128/JCM.02268-10

[8] Boehme, C.C., Nicol, M.P., Nabeta, P., Michael, J.S., Gotuzzo, E., et al. (2011) Feasibility, Diagnostic Accuracy, and Effectiveness of Decentralised Use of the Xpert MTB/RIF Test for Diagnosis of Tuberculosis and Multidrug Resistance: A Multicentre Implementation Study. Lancet, 377, 1495-1505.

http://www.pubmedcentral.nih.gov/articlerender.fcgi?artid=3085933\&tool=pmcentrez\&rendertype=abstract http://dx.doi.org/10.1016/S0140-6736(11)60438-8

[9] Jobbagy, Z., van Atta, R., Murphy, K.M., Eshleman, J.R. and Gocke, C.D. (2007) Evaluation of the Cepheid GeneXpert BCR-ABL Assay. Journal of Molecular Diagnostics, 9, 220-227.

http://www.pubmedcentral.nih.gov/articlerender.fcgi?artid=1867453\&tool=pmcentrez\&rendertype=abstract http://dx.doi.org/10.2353/jmoldx.2007.060112

[10] Small, P.M. and Pai, M. (2010) Tuberculosis Diagnosis-Time for a Game Change. The New England Journal of Medicine, 363, 1070-1071. http://dx.doi.org/10.1056/NEJMe1008496

[11] FIND Welcomes First Phase of Xpert MTB/RIF Buy-Down to Reduce Cost of Cartridges by $40 \%$ for High-Burden Countries (2012). http://www.finddiagnostics.org/resource-centre/news/120823.html

[12] Dye, C. (2012) The Potential Impact of New Diagnostic Tests on Tuberculosis Epidemics. Indian Journal of Medical Research, 135, 737-744. http://www.ncbi.nlm.nih.gov/pubmed/22771607

[13] WHO (2013) Global Tuberculosis Report 2013. Geneva.

[14] WHO (2011) Report 2011: Global Tuberculosis Control. WHO Report, Geneva.

[15] Fortson, J.G. (2011) Mortality Risk and Human Capital Investment: The Impact of HIV/AIDS in Sub-Saharan Africa. The Review of Economics and Statistics, 93, 1-15.

[16] Sommers, M. and Wilson, W. (2011) Governance, Security and Culture: Assessing Africa’s Youth Bulge. International Journal of Conflict and Violence, 5, 345-356.

[17] CIA World Factbook (2012). https://www.cia.gov/library/publications/the-world-factbook/

[18] Singhal, S., Mahajan, S.N., Diwan, S.K., Gaidane, A. and Quazi, Z.S. (2011) Correlation of Sputum Smear Status with CD4 Count in Cases of Pulmonary Tuberculosis and HIV Co-Infected Patients-A Hospital Based Study in a Rural Area of Central India. The Indian Journal of Tuberculosis, 58, 108-112.

[19] Nunn, P., Williams, B., Floyd, K., Dye, C., Elzinga, G. and Raviglione, M. (2005) Tuberculosis Control in the Era of HIV. Nature Reviews Immunology, 5, 819-826. http://dx.doi.org/10.1038/nri1704

[20] Jones-López, E.C., Namugga, O., Mumbowa, F., Ssebidandi, M., Mbabazi, O., Moine, S., et al. (2013) Cough Aerosols of Mycobacterium tuberculosis Predict New Infection: A Household Contact Study. American Journal of Respiratory and Critical Care Medicine, 187, 1007-1015. http://dx.doi.org/10.1164/rccm.201208-1422OC

[21] Reynolds, D.L., Gillis, F., Kitai, I., Deamond, S.L., Silverman, M., King, S.M., et al. (2006) Transmission of Mycobacterium tuberculosis from an Infant. International Journal of Tuberculosis and Lung Disease, 10, 1051-1056. http://www.ncbi.nlm.nih.gov/pubmed/16964800

[22] Carvalho, A.C.C., Riemer, K.D.E., Nunes, Z.B., Martins, M., Comelli, M., Marinoni, A. and Kritski, A.L. (2001) 
Transmission of Mycobacterium tuberculosis to Contacts of HIV-Infected Tuberculosis Patients. American Journal of Respiratory and Critical Care Medicine, 164, 2166-2171. http://dx.doi.org/10.1164/ajrccm.164.12.2103078

[23] Espinal, M.A., Peréz, E.N., Baéz, J., Hénriquez, L., Fernández, K., et al. (2000) Infectiousness of Mycobacterium tuberculosis in HIV-1-Infected Patients with Tuberculosis: A Prospective Study. Lancet, 355, 275-280. http://www.ncbi.nlm.nih.gov/pubmed/23278714

[24] Lin, H., Dowdy, D., Dye, C., Murray, M. and Cohen, T. (2012) The Impact of New Tuberculosis Diagnostics on Transmission: Why Context Matters. Bulletin of the World Health Organization, 1-22.

[25] Menzies, N.A., Cohen, T., Lin, H., Murray, M. and Salomon, J.A. (2012) Population Health Impact and Cost-Effectiveness of Tuberculosis Diagnosis with Xpert MTB/RIF: A Dynamic Simulation and Economic Evaluation. PLoS Medicine, 9, Article ID: e1001347.

[26] Keeling, M. and Rohani, P. (2008) Modeling Infectious Diseases in Humans and Animals. Princeton University Press, Princeton and Oxford.

[27] CIA World Factbook (2013). https://www.cia.gov/library/publications/the-world-factbook/

[28] Ngangro, N.N., Ngarhounoum, D., Ngangro, M.N., Rangar, N., Siriwardana, M.G., des Fontaines, V.H. and Chauvin, P. (2012) Pulmonary Tuberculosis Diagnostic Delays in Chad: A Multicenter, Hospital-Based Survey in Ndjamena and Moundou. BMC Public Health, 12, 513. http://www.ncbi.nlm.nih.gov/pubmed/22776241 http://dx.doi.org/10.1186/1471-2458-12-513

[29] Boehme, C.C., Nicol, M.P., Nabeta, P., Michael, J.S., Gotuzzo, E., Tahirli, R., et al. (2011) Feasibility, Diagnostic Accuracy, and Effectiveness of Decentralised Use of the Xpert MTB/RIF Test for Diagnosis of Tuberculosis and Multidrug Resistance: A Multicentre Implementation Study. Lancet, 377, 1495-1505. http://www.pubmedcentral.nih.gov/articlerender.fcgi?artid=3085933\&tool=pmcentrez\&rendertype=abstract http://dx.doi.org/10.1016/S0140-6736(11)60438-8

[30] WHO (2013) Tuberculosis Fact Sheet No. 104. http://www.who.int/mediacentre/factsheets/fs104/en/index.html

[31] Evans, C.A. (2011) GeneXpert-A Game-Changer for Tuberculosis Control? PLoS Medicine, 8, e1001064. http://dx.doi.org/10.1371/journal.pmed.1001064 
Scientific Research Publishing (SCIRP) is one of the largest Open Access journal publishers. It is currently publishing more than 200 open access, online, peer-reviewed journals covering a wide range of academic disciplines. SCIRP serves the worldwide academic communities and contributes to the progress and application of science with its publication.

Other selected journals from SCIRP are listed as below. Submit your manuscript to us via either submit@scirp.org or Online Submission Portal.
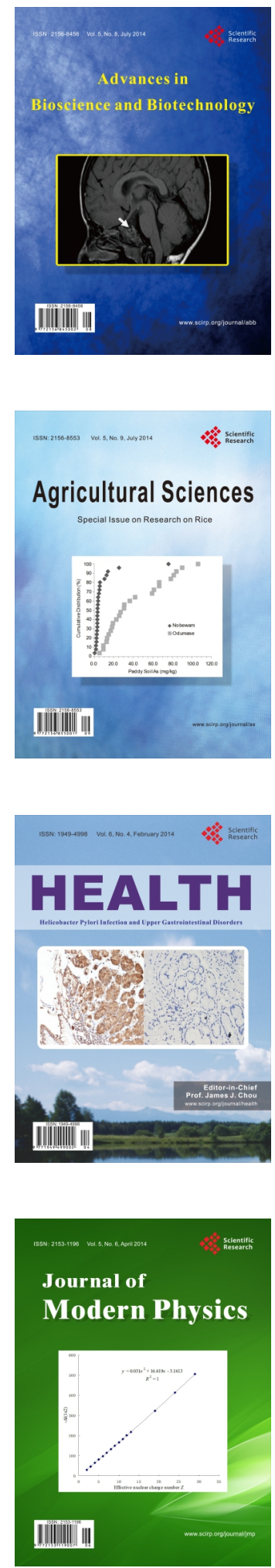
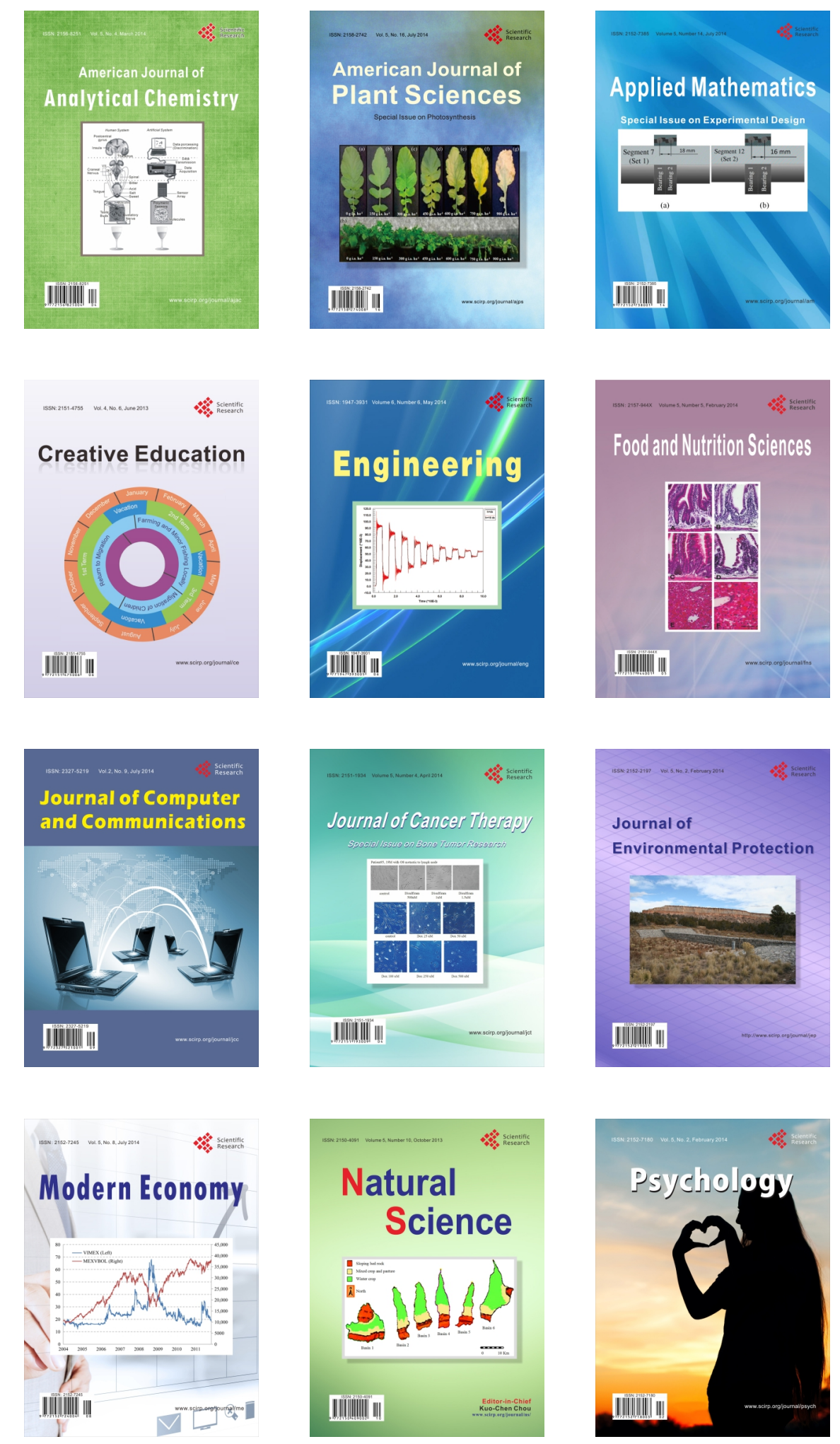